between both groups (mean 2.5 vs $3.1, \mathrm{p}=0.09$ ), however, the outcome was trending to be worse in MCA occlusion patients, this could be the result of the relatively higher rate of procedural complications in MCA occlusions, $18.5 \%$ in MCA (24/130 cases, 13 distal embolic, $8 \mathrm{SAH} / \mathrm{sICH}, 3$ arterial dissections) versus $12.5 \% \quad(7 / 55$ cases, 4 distal embolic, $3 \mathrm{SAH} / \mathrm{sICH})$.

Conclusions Significant procedural difficulty is encountered during ET of ICA relative to MCA occlusions, requiring increased procedural time and passes. Although final successful recanalization rates are equivalent, we noted a paradoxical trend for less functional independence with MCA occlusions this could be due to the higher rate of complications in the MCA cohort, and the better collaterals through the circle of Willis in the ICA cohort.

Disclosures M. Aly: None. R. Abdalla: None. M. Hurley: None. A. Shaibani: None. S. Ansari: None.

\section{E-085 MULTICENTER RETROSPECTIVE STUDY OF THE SOFIA FOR ASPIRATION IN ACUTE ISCHEMIC STROKE (MRS SOFIA)}

${ }^{1} W_{\text {Brinjikji }}{ }^{2}{ }^{2} \mathrm{R}$ Deleacy, ${ }^{3} \mathrm{D}$ Meila, ${ }^{4} \mathrm{M}$ Mokin, ${ }^{5} \mathrm{E}$ Samaniego, ${ }^{6} \mathrm{~A}$ Cora, ${ }^{7} \mathrm{M}$ Shapiro, ${ }^{7} \mathrm{~S}$ Agarwal, ${ }^{7} \mathrm{D}$ Bageac, ${ }^{5} \mathrm{~J}$ Roa, ${ }^{4} \mathrm{Z}$ Ren, ${ }^{4} \mathrm{~W}$ Li, ${ }^{8} \mathrm{~L}$ Rinaldo, ${ }^{1} \mathrm{H}$ Cloft, ${ }^{6} \mathrm{D}$ Volders, ${ }^{6} \mathrm{~T}$ Huynh, ${ }^{7}$ E Raz. ${ }^{1}$ Radiology, Mayo Clinic, Rochester, MN; ${ }^{2}$ Radiology, Mt. Sinai, New York, NY; ${ }^{3}$ Radiology, Hannover Medical Center, Hannover, GERMANY; ${ }^{4}$ Neurology, University of South Florida, Tampa, FL; ${ }^{5}$ Neurology, University of lowa, Hawkins City, IA; ${ }^{6}$ Radiology, Dalhousie, Halifax, NS, CANADA; 'Radiology, NYU Medical Center, New York, NY; ${ }^{8}$ Neurosurgery, Mayo Clinic, Rochester, MN

\subsection{6/neurintsurg-2020-SNIS.119}

Background and Purpose There are a myriad of aspiration catheters used for primary aspiration for mechanical embolectomy for acute ischemic stroke. Over the past several years there has been increased interest in the use of the 6Fr Sofia Plus aspiration catheter as a primary aspiration catheter due to its trackability and navigability. We performed a multicenter retrospective study examining the efficacy of the Sofia aspiration catheter as a stand-alone aspiration treatment for large vessel occlusion.

Materials and Methods This was an investigator initiated study and no study funding was received. Following Institutional Review Board Approval, consecutive cases in which the Sofia catheter was used for aspiration thrombectomy for treatment of large vessel occlusion were included. Exclusion criteria were the following: 1) Sofia not used for first pass, and 2) stent-triever used as an adjunct on the first pass. The primary outcome of the study was first pass recanalization (TICI 2b/3). Secondary outcomes included first pass TICI $2 \mathrm{c} / 3$, final TICI using Sofia alone, cross-over to other thrombectomy devices, number of passes, time from puncture to recanalization and complications.

Results From the six centers, a total of 323 patients were included. First pass recanalization with TICI 2b/3 was achieved in $69.7 \%$ of cases. First pass recanalization with TICI $2 \mathrm{c} / 3$ was achieved in $48.9 \%$ of cases. $74.8 \%$ patients achieved a TICI $2 \mathrm{~b} / 3$ with the Sofia alone. Cross-over to other thrombectomy devices occurred in $29.1 \%$ of cases. The median number of passes performed was 1.5 (IQR $=1-3)$. Median time from puncture to recanalization was 25 $(\mathrm{IQR}=15-35)$. Procedure related complications occurred in $2.8 \%$ of cases.
Conclusion Our study highlights the potential advantage of the $5 \mathrm{Fr}$ and $6 \mathrm{Fr}$ Sofia aspiration catheters for primary aspiration thrombectomy in acute ischemic stroke. We were able to achieve high rates of first pass recanalization with low cross-over rates to other thrombectomy devices. Median procedure time was low as were procedural complications.

Disclosures W. Brinjikji: 1; C; NIH/NINDS, Cerenovus. 2; C; Microvention, Cerenovus. 4; C; Marblehead Medical LLC. R. DeLeacy: None. D. Meila: None. M. Mokin: None. E. Samaniego: None. A. Cora: None. M. Shapiro: None. S. Agarwal: None. D. Bageac: None. J. Roa: None. Z. Ren: None. W. Li: None. L. Rinaldo: None. H. Cloft: None. D. Volders: None. T. Huynh: None. E. Raz: None.

\section{E-087 SAFETY AND EFFICACY OF TRACSTAR LARGE DISTAL PLATFORM DURING MECHANICAL THROMBECTOMY}

D Bageac*, R De Leacy. Neurosurgery, The Mount Sinai Hospital, New York, NY

\subsection{6/neurintsurg-2020-SNIS.120}

Introduction Mechanical thrombectomy is the treatment of choice for ischemic stroke due to large vessel occlusion. One common reason for failure or prolonged procedure time is vascular touristy inhibiting access to the site of occlusion. A novel device -the TracStar Large Distal Platform (LDP) -may offer improved navigation of tortuous vasculature as well as more distal final positioning when compared to commonly used guide catheters. We investigate the safety and efficacy of the TracStar LDP when used during mechanical thrombectomy.

Methods We perform a multicenter retrospective review of mechanical thrombectomy procedures during which the TracStar LDP was utilized. Procedural information, complications, and key timepoints (groin puncture, clot-first-touch, etc.) were recorded as detailed in the operative note. Vascular tortuosity was assessed via pre-procedural CTA. Distal-most position achieved with TracStar was determined by review of intraprocedural imaging. Linear regression was performed to investigate the potential relationship between vascular tortuosity and procedure time.

Results In a preliminary analysis of 20 cases, successful reperfusion (TICI $2 \mathrm{~b} / 3$ ) was achieved in $88 \%$ of cases, requiring 1.8 passes on average (rage 1-5). Average NIHSS at presentation was $17.8 \pm 6$. Anterior circulation emboli were encountered in 15 cases. Average time to clot-first touch was $12 \pm 7$ minutes and average time to final reperfusion was $50 \pm 27.6$ minutes. Good functional outcome (mRS 0-2) at 90 days was achieved in $35 \%$ of cases. There was no association between vascular tortuosity and procedure time. There were no procedural complications, and TracStar LDP was exchanged for an alternative guide catheter in two cases due to failure to maintain positing.

Conclusion Mechanical thrombectomy with the Tracstar LDP is safe and effective. Access beyond the horizonal petrous segment of the ICA was achieved in the majority of cases. Interestingly, ICA tortuosity was not associated with an increased time to clot-first-touched. Final analysis of 30 patients in this multi-center retrospective study will be available for presentation at the SNIS Annual Meeting 2020 .

Disclosures D. Bageac: None. R. De Leacy: None. 